\title{
Diagnostic de la constipation chez l'enfant
}

\author{
Frédérique Sauvat
}

Service de Chirurgie pédiatrique, UFR Necker-Enfants Malades, Université René Descartes Paris V, Paris, France

\section{Mots-clés}

Constipation • Maladie de Hirschsprung • Pédiatrie

\section{Résumé}

La constipation est un motif fréquent de consultation d'un pédiatre. A titre de symptôme, une constipation peut être due à des etiologies diverses en termes de fréquence, de nature et de gravité. La constipation peut être d'origine médicale ou chirurgicale, ou idiopathique. Les étiologies chirurgicales sont principalement représentées par la maladie de Hirschsprung. II s'agit du principal diagnostic différentiel même s'il est 50 fois moins fréquent que la constipation idiopathique ou fonctionnelle. Une anamnèse détaillée et un examen clinique, incluant un examen anal, sont les meilleurs moyens de parvenir à un diagnostic. Aucun autre examen n'est nécessaire dans la plupart des cas et toutes les explorations doivent être gérées en fonction des considérations cliniques.

Copyright $\odot 2007$ Nestec Ltd., Vevey/S. Karger AG, Basel

La constipation est le motif de consultation d'un pédiatre dans $3 \%$ des cas et d'un gastro-entérologue dans plus de $25 \%[1,2]$. La première difficulté concernant la constipation consiste à définir le nombre normal de selles qu'un enfant doit émettre. Ce nombre varie en effet en fonction de l'âge et du type d'alimentation. La constipation peut se définir par une fréquence des selles spontanées inférieure à 3 par semaine. Elle peut s'accompagner d'autres signes (douleurs abdominales) plus ou moins in-

\section{KARGER}

Fax +4161306 1234

E-Mail karger@karger.ch

www.karger.com
(C) 2007 Nestec Ltd., Vevey/S. Karger AG, Basel

0250-9644/07/0652-0063\$23.50/0

Accessible en ligne à: www.karger.com/anf validants et retentissant sur la vie sociale et scolaire [3]. Comme symptôme, la constipation peut relever d'étiologies diverses en termes de fréquence, de nature et de gravité [4]. Ces étiologies peuvent être divisées en causes médicales et chirurgicales, la constipation étant le plus souvent idiopathique ou fonctionnelle [5].

\section{Constipation idiopathique}

Il s'agit de loin de l'étiologie la plus fréquente d'une constipation [6]. Avec la croissance de l'enfant, des modifications physiologiques normales surviennent dans l'intestin et le côlon et diminuent le nombre quotidien de selles d'une moyenne de 2,2 chez les nourrissons de moins d'un an à 1,4 chez les enfants d'un an [7]. La constipation peut être définie comme l'émission de moins d'une selle par jour au cours des premiers mois de la vie et de moins de trois par semaine chez les enfants plus âgés. Le pic de fréquence de la constipation correspond à l'âge de l'apprentissage de la propreté (2-4 ans), avec une prévalence plus élevée chez les garçons. Le diagnostic de constipation fonctionnelle peut reposer sur les critères de Rome, en fonction de l'âge du patient $[2,8]$.

Le diagnostic est confirmé quand au moins deux des signes suivants persiste pendant au moins un mois: (1) deux selles ou moins par semaine; (2) au moins un épisode d'incontinence par semaine; (3) antécédent de comportement de rétention de selles; (4) douleurs abdominales; (5) fécalome intrarectal, et (6) selles volumineuses pouvant obturer les toilettes. 
Environ $40 \%$ des enfants souffrant d'une constipation fonctionnelle en présentent les signes au cours de la première année de leur vie. Il semble également que, chez les enfants âgés de moins de 4 ans, le pronostic soit meilleur si un traitement est instauré avant l'âge de 2 ans. Des épisodes d'incontinence sont présents chez $84 \%$ des patients [9]. Dans les cas typiques, aucune exploration complémentaire n'est nécessaire et une radiographie abdominale sans préparation est suffisante pour l'évaluation de la rétention fécale si l'examen clinique est difficile (obésité) [10]. Il est nécessaire de confirmer l'absence d'anomalies vertébrales ou médullaires (voir plus bas). De la même façon, un toucher anorectal doit être effectué afin d'éliminer une malformation anorectale minime, d'examiner la tonicité du sphincter anal et de rechercher la présence d'un fécalome [11]. Le traitement repose sur une évacuation aussi complète que possible du côlon, un ramollissement des selles et des modifications des apports alimentaires et du mode de vie. Des erreurs diététiques (déshydratation, régime pauvre en résidus, etc.) ainsi que des causes locales peuvent perpétuer une constipation tout en rendant l'expulsion des selles difficile ou douloureuse.

\section{Causes médicales de constipation}

Dans des cas plus rares, la constipation chez l'enfant est due à un dysfonctionnement endocrine ou à une cause métabolique ou toxique.

L'hypothyroïdie est la principale cause endocrine de constipation. Un dépistage systématique au cours de la période néonatale (en association au test de Guthrie) permet toutefois un diagnostic précoce, réduisant ainsi les conséquences neurologiques d'un diagnostic plus tardif. Le diabète [12] et l'hypoparathyroïdie ont été également décrits comme causes de constipation, mais des troubles $\mathrm{du}$ transit sont rarement le premier signe de ces affections.

Des déséquilibres électrolytiques peuvent ralentir les mouvements coliques, comme en cas d'hypo et d'hypercalcémie $[13,14]$.

La possibilité d'une intoxication doit toujours être envisagée lors du recueil des antécédents médicaux, sans oublier tout traitement reçu par la mère qui allaite. Les principaux traitements responsables d'une constipation au cours de la petite enfance sont les anticholinergiques, les antispasmodiques et certaines résines (cholestyramine). L'éventuelle responsabilité de l'ajout d'un épaississant (Gumilk ${ }^{\circledR}$ ) aux formules pour nourrissons ne doit pas être oubliée car pouvant être à l'origine d'une consti- pation, et même d'un authentique syndrome occlusif dû à l'accumulation de selles compactes, particulièrement dans le caecum.

Comme déjà mentionné, un régime alimentaire inadéquat peut induire une constipation. Les principales étiologies sont les suivantes: (1) malnutrition, anorexie; (2) déshydratation [15]; (3) apports excessifs en lait de vache [16], et (4) insuffisance en résidus, particulièrement chez les enfants les plus grands.

Une maladie cœliaque [17] (intolérance au gluten) peut s'accompagner d'épisodes de constipation.

Une mucoviscidose peut s'accompagner d'une constipation au cours de la période néonatale (iléus méconial) ou plus tard au cours de la petite enfance, en raison d'une insuffisance pancréatique exocrine. Au cours de la période prénatale, une mucoviscidose peut être suspectée quand un syndrome occlusif est associé à une hyperéchogénicité digestive (et aboutit au diagnostic prénatal par amniocentèse). A la naissance, un iléus méconial est une occlusion de l'iléon avec élimination retardée du méconium et distension abdominale. Laspect radiographique est typique quand des opacités sont présentes dans la partie droite de l'abdomen. Une injection rectale de gastrograffine révèle un côlon non fonctionnel et un aspect typique dans le caecum et l'iléon où le produit forme de petites boules de méconium. En cas d'intervention, qui doit être évitée par tout moyen, l'aspect macroscopique pâteux du méconium est caractéristique. La trypsine immunoréactive et la biologie moléculaire confirment le diagnostic. Comme dans le cas de l'hypothyroïdie, un dépistage est désormais obligatoire en France au cours de la période néonatale.

\section{Causes chirurgicales de constipation}

La principale cause organique de constipation est la maladie de Hirschsprung. Il ne faut cependant pas oublier que celle-ci est 50 fois moins fréquente que la constipation idiopathique.

\section{Maladie de Hirschsprung}

La maladie de Hirschsprung, ou mégacôlon congénital, est une malformation congénitale caractérisée par l'absence de cellules ganglionnaires entériques sur une longueur variable de l'intestin. Quelle que soit la longueur de la région atteinte, l'innervation intrinsèque intestinale débute toujours au-dessus de la région anale et s'étend plus ou moins jusqu'au côlon ou même jusqu'à l'intestin grêle. Dans $75-80 \%$ des cas, la maladie de 
Hirschsprung affecte le rectum et le sigmoïde [18]. La forme colique totale est présente dans $10 \%$ des cas, et une aganglionose intestinale totale (incluant le jéjunum) dans moins de $1 \%$. L'anomalie est due à l'absence de cellules ganglionnaires dans les plexus myentériques et sous-muqueux de Meissner et Auerbach, et est associée à une hypertrophie de fibres amyéliniques. Cette aganglionose est responsable de l'hypertonicité de l'intestin pathologique.

\section{Épidémiologie de la maladie de Hirschsprung}

La fréquence de la maladie de Hirschsprung dans la population générale est de 1 pour 5000 naissances [19]. Elle s'observe principalement chez les garçons: 4/1 pour la forme courte (recto-sigmoïde). Le risque de survenue chez les parents du premier degré est faible (4\%) mais augmente significativement quand le cas index est de sexe féminin ou présente une forme étendue. La plupart des cas sont sporadiques; la forme familiale représente $6-15 \%$ des cas avec transmission autosomique dominante et pénétrance incomplète [20-22].

\section{Présentation clinique}

La symptomatologie varie en fonction de l'âge et de l'étendue de la maladie. Une obstruction fonctionnelle est présente dans tous les cas.

Diagnostic néonatal d'une maladie de Hirschsprung non compliquée $[23,24]$

La possibilité d'une maladie de Hirschsprung est systématiquement suspectée chez tout nouveau-né présentant une obstruction fonctionnelle, particulièrement s'il est né à terme et si l'échographie prénatale était normale.

Les signes cliniques habituels sont les suivants: (1) retard de l'émission de méconium, qui survient après 2448 heures; (2) augmentation progressive d'une distension abdominale au cours des deux premiers jours de la vie, et (3) vomissements alimentaires, des vomissements bilieux sont souvent plus tardifs.

Le meilleur signe clinique pour un diagnostic positif de maladie de Hirschsprung est l'émission subite de gaz et de méconium après un toucher rectal ou la mise en place d'une sonde rectale. Dans la forme recto-sigmoïde, la sonde rectale, placée au-dessus de la partie aganglionnaire, permet l'évacuation des selles retenues et une diminution rapide de la distension abdominale [25]. La sonde doit être mise en place après une radiographie (pas de pneumopéritoine) et il faut veiller à éviter une perforation, particulièrement en cas de suspicion d'une entéro- colite. L'absence d'émission subite n'exclut pas le diagnostic dans le cas d'une forme longue. Le diagnostic de maladie de Hirschsprung est posé dans la majorité des cas au cours du premier trimestre [26].

Diagnostic de la maladie de Hirschsprung chez le nourrisson

La possibilité d'une maladie de Hirschsprung chez un nouveau-né présentant une constipation doit être impérativement suspectée, particulièrement si le trouble de la motricité est apparu ou s'est aggravé après le sevrage et la diversification alimentaire, moment où les selles deviennent compactes. La fréquence des selles est variable, parfois avec une absence de défécation pendant plusieurs jours. L'abdomen présente une distension chronique, qui diminue toutefois après une stimulation (laxatif, etc.). L'examen rectal élimine une malformation anorectale basse (sténose anale in situ) et facilite la détection d'une impaction fécale haute. La croissance est habituellement médiocre.

\section{Diagnostic tardif}

Les formes courtes de la maladie de Hirschsprung peuvent être diagnostiquées dans la quasi-totalité des cas. Ces enfants ont de longs antécédents de constipation opiniâtre avec épisodes récidivants de sub-occlusion et distension de la totalité de la paroi abdominale. La constipation est connue à partir de la naissance, avec des exonérations stimulées uniquement à intervalles de plusieurs jours. Des selles souvent desséchées sont mélangées à des selles liquides et d'odeur fétide due à une rétention chronique. Un point clinique important est que la continence est toujours normale dans la maladie de Hirschsprung; une encoprésie n'est jamais associée à cette maladie. Dans certains cas, la maladie de Hirschsprung a un impact important sur la croissance et le développement psychologique à long terme [27].

Maladie de Hirschsprung étendue [28, 29]

Le diagnostic de la forme longue de la maladie de Hirschsprung est souvent difficile et tardif [30]. Le tableau clinique peut être très variable: (1) occlusion intestinale néonatale (iléale) parfois incomplète avec ou sans évacuation spontanée de méconium, et des signes cliniques, tels qu'une radiographie, pourraient aider à diagnostiquer un iléus méconial; (2) entérocolite néonatale $[31,32]$, qui pourrait être liée à la maladie de Hirschsprung étendue en l'absence de prématurité ou de cardiopathie sévère; (3) présentation aiguë avec perforation appendiculaire ou caecale [33], et (4) constipation sévère 

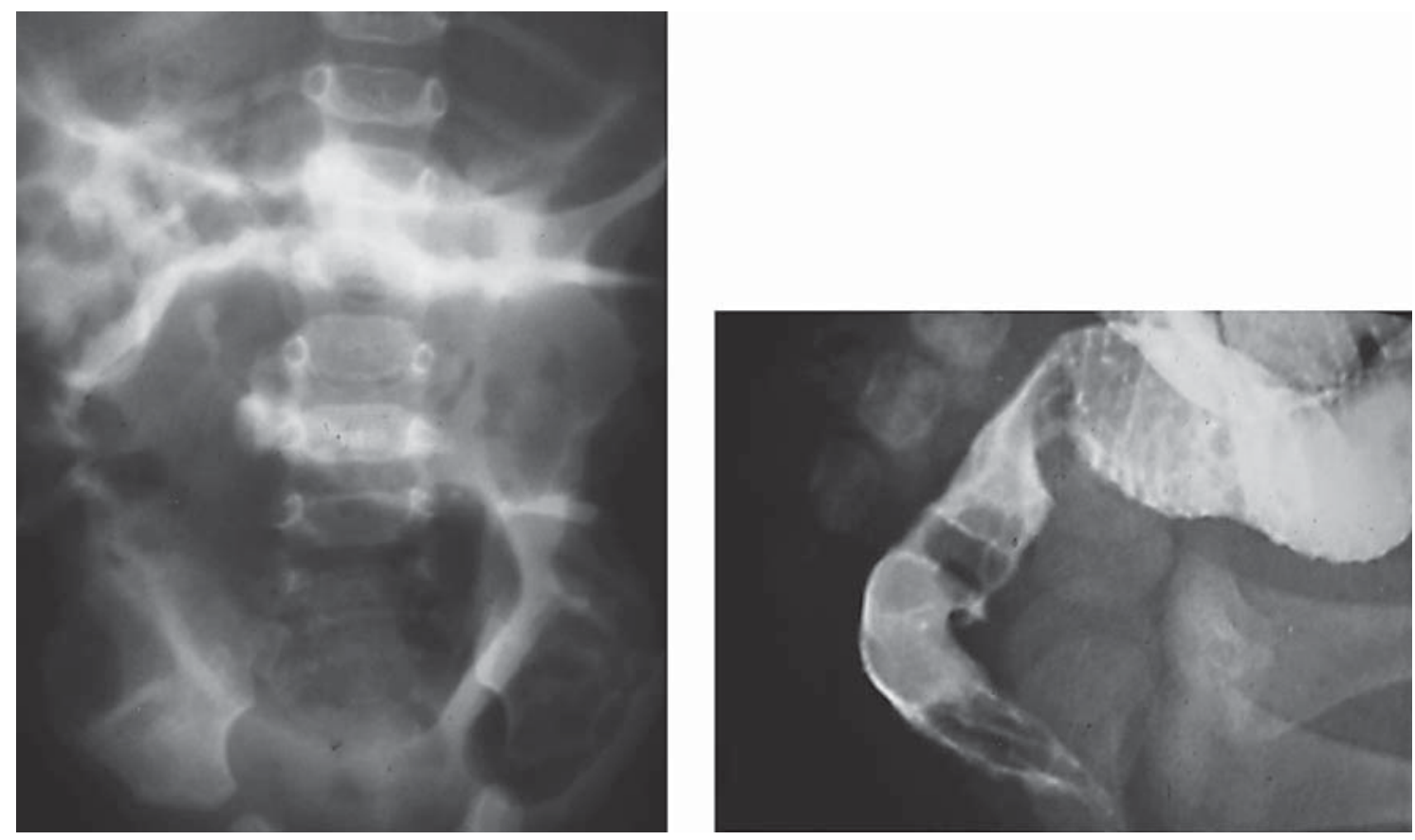

Fig. 1. Aspect typique de maladie de Hirschsprung recto-sigmoïdienne à la radiographie et au lavement baryte.

chez un nouveau-né, particulièrement due à une mauvaise tolérance avec un effet majeur sur la croissance.

\section{Maladie de Hirschsprung compliquée}

Les deux principales complications de la maladie de Hirschsprung sont une perforation intestinale et une entérocolite. Ces complications peuvent révéler la maladie ou survenir au cours de celle-ci et engager le pronostic vital.

Une entérocolite [34] peut être considérée comme une septicémie d'origine intestinale, secondaire à une prolifération microbienne endoluminale induite par une stase associée à une ulcération de la paroi intestinale et à une ischémie par distension. Au plan clinique, l'enfant présente une distension abdominale douloureuse majeure et un sepsis profond. Les selles sont liquides et d'odeur fétide ou sanglantes. La radiographie révèle une dilatation globale, mais une pneumatose est rare. Le traitement de l'entérocolite due à une maladie de Hirschsprung repose sur une antibiothérapie à large spectre (centrée sur les germes Gram-négatif et anaérobies) et sur une dérivation chirurgicale (dans une zone normalement innervée).

Une perforation peut survenir dans une zone normalement innervée [35]. Une chirurgie d'urgence est nécessaire en cas de pneumopéritoine. Un diagnostic positif de maladie de Hirschsprung pourrait être difficile car une disparité de calibre peut être absente. Dans une situation de ce type, le diagnostic doit être conservé à l'esprit et aboutir à une biopsie rectale en association à une stomie au-dessus de la perforation [36].

\section{Diagnostic de la maladie de Hirschsprung}

Le diagnostic de la maladie de Hirschsprung repose sur l'opacification colique, mais principalement sur une biopsie rectale qui est la seule façon de poser un diagnostic net en l'absence de cellules ganglionnaires.

Opacification radiologique $[37,38]$

L'objectif de l'opacification colique est de montrer la disparité de diamètre entre la partie aganglionnaire rétrécie et le segment normal dilaté. De plus, elle permet l'estimation de la longueur du côlon atteint. Cet examen est souvent d'interprétation difficile chez le nouveau-né.

Il doit être effectué dans de bonnes conditions et en l'absence d'un pneumopéritoine et d'une entérocolite. La progression du produit de contraste (préférentiellement la baryte) est suivie sous scopie, avec incidences de face et de profil et centrage sur la disparité des diamètres. Les meilleurs clichés sont souvent les images sans préparation (et au bout d'1 heure). 
Dans la forme recto-sigmoïdienne, la partie rectale présente un aspect normal, suivie par une partie transitionnelle s'élargissant jusqu'au côlon normal dilaté. Cette disparité de calibre est spécifique de la maladie de Hirschsprung (fig. 1).

Dans la forme colique totale, l'opacification peut présenter différents aspects [39]: (1) un côlon court en forme de point d'interrogation, avec disparition des angles coliques droit et gauche (fig. 2); (2) un microcôlon associé à une dilatation plus ou moins importante de l'iléon - dans cette forme, le diagnostic différentiel est un iléus méconial ou un syndrome microcôlon mégavessie, et (3) un aspect normal (30\%).

\section{Manométrie anorectale [40,41]}

À la manométrie, l'absence de réflexe inhibiteur rectoanal est le principal signe pour le diagnostic de maladie de Hirschsprung. Toutefois, la présence d'un réflexe faussement positif rend une biopsie rectale essentielle. On considère habituellement que la manométrie a peu d'intérêt chez le nouveau-né (avant le $3^{\text {ème }}$ mois de la vie).

\section{Biopsie rectale}

Un examen histologique permet un diagnostic définitif et est donc capital. La biopsie rectale doit être effectuée au moins $3 \mathrm{~cm}$ au-dessus de la marge anale afin d'éviter un résultat faussement positif (une biopsie effectuée en position trop basse pourrait être située dans une partie hypoganglionnaire normale). Cette biopsie peut être effectuée de deux façons.

(1) Une biopsie par aspiration rectale (à la pince de $\mathrm{H}$. Noblett), réalisée sans anesthésie. Habituellement, deux fragments sont prélevés à 3 et $6 \mathrm{~cm}$ de la marge anale. Cette technique présente des limites: un anatomopathologiste entraîné est nécessaire et l'échantillon biopsique prélevé doit contenir de la muscularis mucosae et, si possible, de la sous-muqueuse.

(2) Une biopsie chirurgicale, qui doit contenir la totalité de la paroi rectale (technique de Swenson). Elle nécessite une anesthésie générale mais permet une biopsie plus profonde et plus informative.

Les résultats histologiques en faveur d'un diagnostic de maladie de Hirschsprung sont les suivants [42]: (1) à l'histologie standard et sur pièces fixées, absence de cellules ganglionnaires et hypertrophie des fibres amyéliniques (souvent absente chez le nouveau-né) [43], et (2) l'examen immunohistochimique (pièces congelées) montre une hyperplasie des fibres cholinergiques dans les parties sous-muqueuses et musculeuses, ainsi que des fibres nerveuses remontant dans la muqueuse.

Constipation chez l'enfant

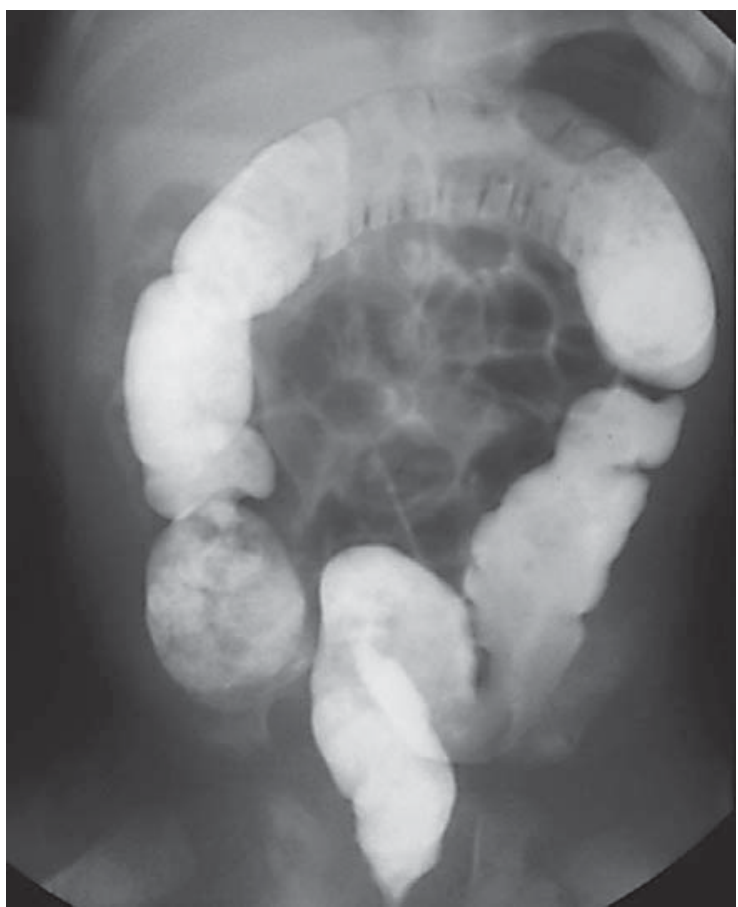

Fig. 2. Opacification radiologique dans la forme colique de la maladie de Hirschsprung.

\section{Traitement}

Le traitement de la maladie de Hirschsprung est chirurgical et consiste en une exérèse de la zone aganglionnaire et en une anastomose entre l'anus et le côlon normal innervé [44]. Au cours de la période préopératoire, les selles doivent être évacuées par évacuation rectale ou stomie.

\section{Prise en charge préopératoire}

Une évacuation rectale est utile en éliminant les selles par une sonde rectale poussée au-dessus du côlon aganglionnaire. Le tube doit être mis en place une à trois fois par jour, permettant une diminution de la distension abdominale et une correction de la courbe de croissance. Cette approche simple ne peut être envisagée que pour la forme courte de la maladie de Hirschsprung. Elle permet au patient de revenir à son domicile et d'attendre l'intervention chirurgicale, si la famille est apte à le prendre en charge et à déterminer si l'évacuation rectale est inefficace ou si l'abdomen est distendu.

Stomie

En règle générale, une stomie doit être effectuée dans une partie colique normalement innervée et en position 
aussi distale que possible, en évitant la partie transitionnelle. La stomie sera retirée au cours de l'intervention chirurgicale finale. Les indications d'une stomie au moment du diagnostic sont la forme longue, la forme courte avec évacuation rectale inefficace et la forme compliquée (entérocolite).

$\mathrm{Au}$ cours de l'intervention, une collaboration avec un anatomopathologiste confirme que la stomie a été effectuée en un emplacement correct. Dans la forme longue, les biopsies doivent être nombreuses, et concerner notamment l'appendice, et une iléostomie est souvent nécessaire.

Abaissement du côlon sain

Trois techniques fréquemment employées ont été respectivement décrites par Swenson, Duhamel et Soave. Ces approches peuvent être utilisées avec des résultats équivalents à court et long terme quelle que soit la forme de la maladie et la longueur de l'atteinte. Les innovations chirurgicales incluent l'utilisation de la laparoscopie pour une biopsie ou une dissection intra-abdominale et un abaissement par voie transanale.

Quelle que soit la technique, il est important de se centrer sur les points suivants: (1) préparation préopératoire du côlon; (2) analyse histologique parfaite au cours de l'intervention afin d'éviter un abaissement dans la partie transitionnelle, et (3) antibioprophylaxie.

Certaines complications apparaissent peu après la chirurgie: (1) Fistule de l'anastomose révélée par un abcès pelvien, nécessitant une antibiothérapie et souvent une stomie. (2) Une sténose de l'anastomose est fréquente et habituellement bénigne, avec de bons résultats après dilatation [45]. (3) Une entérocolite demeure une complication plus grave et peut survenir jusqu'à 2 ans après l'intervention [46]. Comme précédemment décrit, les signes cliniques sont une distension abdominale, une diarrhée et une altération de l'état général. (4) Une érosion cutanée périanale s'observe dans la quasi-totalité des cas et répond de façon variable à un traitement local. (5) Des troubles de la défécation peuvent apparaitre lors du suivi à long terme, même sans complication chirurgicale [47].

\section{Techniques chirurgicales}

Technique de Duhamel. Cette technique a été décrite en 1956 et repose sur une conservation rectale utilisant un abaissement rétrorectal du côlon normalement innervé. Elle demeure intéressante en raison de sa simplicité chirurgicale et de la dissection limitée des structures (nerfs pelviens) périrectales. Une anastomose peut être simultanément réalisée au moyen d'agrafes automatiques telles que les GIA. La principale complication de la technique de Duhamel est un éperon colorectal responsable d'un fécalome [48, 49].

Technique de Swenson. Décrite en 1948, cette intervention comporte une résection complète du segment aganglionnaire et une anastomose anale. Les deux principales complications sont une altération neurologique de la vessie (lésion de nerfs pelviens) et une sténose de l'anastomose.

Technique de Soave. Décrite en 1963, le principe est un abaissement dans un cylindre musculaire rectal. L'avantage est l'absence de dissection pelvienne, mais la complication demeure une constipation quand le cylindre est trop long.

Abaissement transanal [50]. Le principe est la réalisation de la technique de Soave sans laparotomie. Cette méthode est particulièrement intéressante chez le nouveauné, mais doit être réservée à la forme courte [51].

Laparoscopie. Une laparoscopie peut être utilisée afin d'effectuer des biopsies ou pour une dissection au cours des interventions de Duhamel ou Swenson.

\section{Autres obstructions fonctionnelles}

Face à une distension abdominale avec émission retardée de méconium chez un nouveau-né, d'autres causes d'obstruction organique ou fonctionnelle doivent être éliminées: imperforation anale, atrésie colique ou iléale (occasionnellement observée avec une hernie de Morgagni), iléus méconial (associé à une mucoviscidose; fig. 3), syndrome du petit côlon gauche ou bouchon de méconium [52].

Les syndromes du petit côlon gauche et du bouchon méconial se caractérisent par le petit diamètre du rectosigmoïde et du côlon gauche jusqu'à l'angle gauche et au sigmoïde ou au côlon iliaque. Au cours de l'opacification, un bouchon est éliminé à la suite d'une injection rectale (la tête de ce moule est souvent grisâtre et le reste du méconium est normal). Le transit est alors strictement normal. Il est toutefois nécessaire de réexaminer ces enfants au cours des premiers mois de vie et d'effectuer une biopsie rectale afin d'exclure toute suspicion de maladie de Hirschsprung [53].

\section{Pseudo-obstruction intestinale chronique}

Le syndrome de pseudo-obstruction intestinale chronique (POIC) est une étiologie très rare d'une constipation. Ce diagnostic peut être discuté dans des cas sévères de constipation et d'échec du traitement médical.

Une POIC se caractérise par des épisodes répétés ou des signes et symptômes continus d'obstruction colique, 


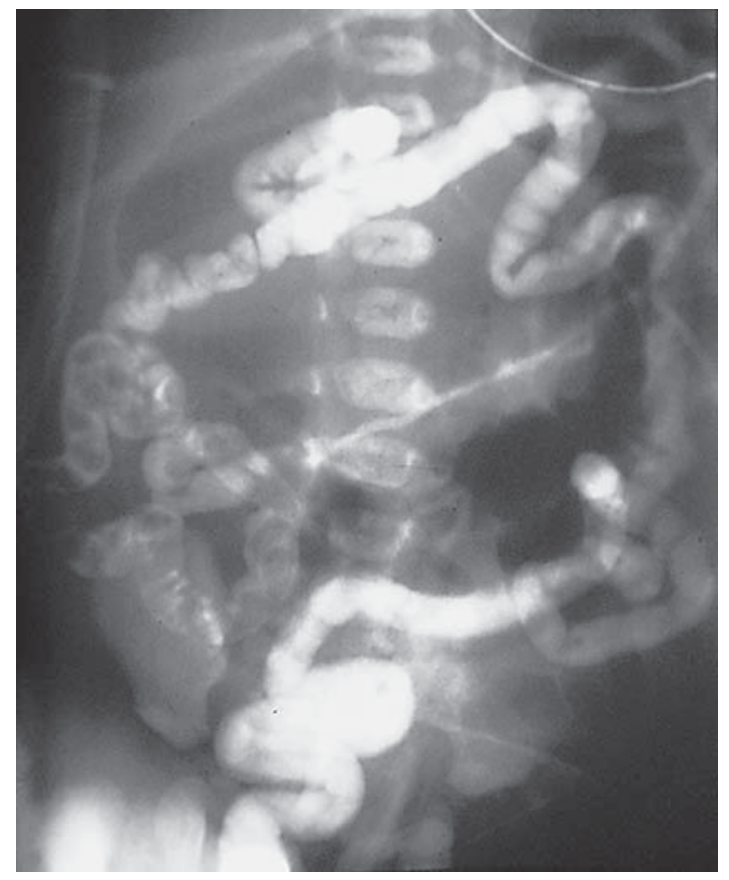

Fig. 3. Aspect typique d'un iléus méconial dans la mucoviscidose, à la naissance.

et en particulier la preuve radiographique d'un côlon dilaté (fig. 4), en l'absence d'une lésion fixée oblitérant la lumière [54].

Quel que soit son type histologique (myogène ou neurogène) [55], un POIC comporte toujours des altérations de la fonction contractile des muscles lisses aboutissant à des anomalies du péristaltisme intestinal et enfin à des troubles nutritionnels dus à la fois à une insuffisance intestinale et à des sepsis récurrents. Des uropathies d'accompagnement doivent être recherchées chez tous les patients présentant un POIC. L'impact clinique de ces uropathies (mégavessie, dilatation rénale, etc.) peut être important, nécessitant parfois une vésicostomie. Cette association explique la nécessité d'une échographie urologique quand le diagnostic étiologique d'une constipation est difficile. De même, une biopsie rectale et une manométrie anorectale peuvent être nécessaires afin de montrer la présence de cellules ganglionnaires et d'un réflexe recto-anal.

\section{Malformations anorectales}

L'examen anal à la naissance est essentiel afin de vérifier la perméabilité rectale. Particulièrement chez les filles (fig. 5), des malformations anorectales avec fistule

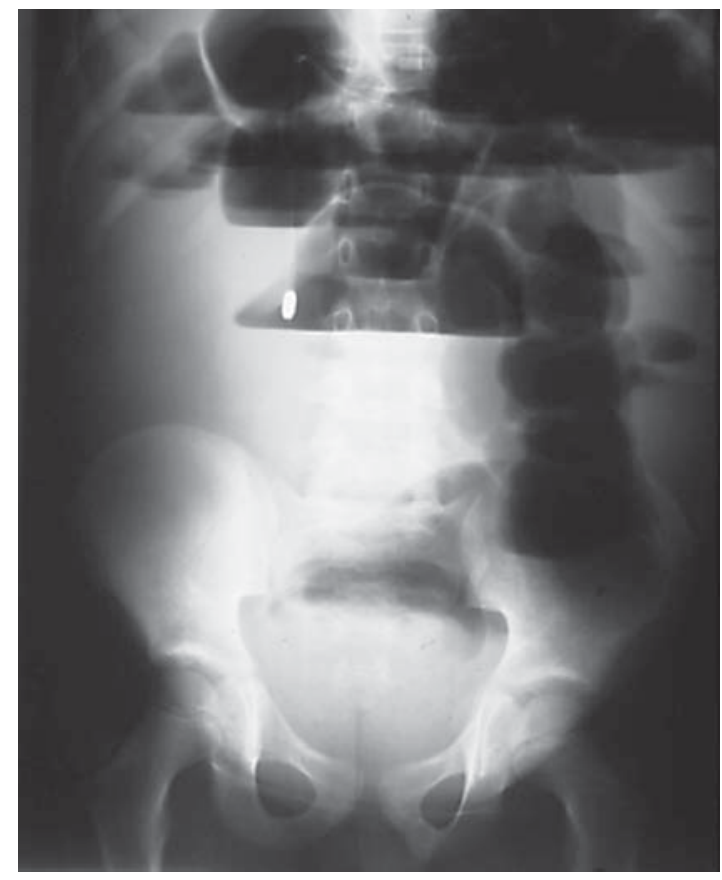

Fig. 4. Dilatation de l'intestin grêle dans un cas de POIC.

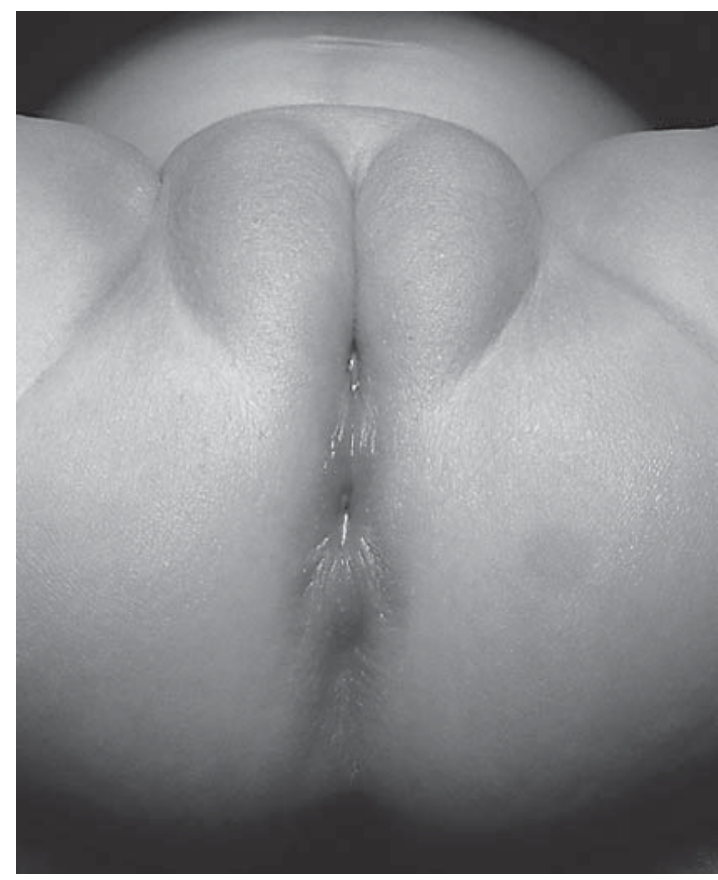

Fig. 5. Malformation anorectale basse chez une fille (anus en position vulvaire). 
périnéale peuvent ne pas être décelées pendant plusieurs mois ou années, bien que le diagnostic soit clinique. L'anus est situé à proximité de la région vulvaire ou scrotale. Le sphincter externe est situé en arrière de la fistule anale. Un toucher rectal est impossible, même avec l'auriculaire.

Comme déjà précisé, au moins un examen anal bien conduit est toujours nécessaire pour le diagnostic d'une constipation.

Une constipation est également fréquente après le traitement de malformations anorectales [56]. Le risque augmente en cas de forme anorectale haute associée à des anomalies sacrées et médullaires [57]. Par exemple, la continence est considérée comme socialement normale dans $75-85 \%$ des cas de malformations basses et dans seulement $50 \%$ après traitement de malformations anorectales hautes. La prise en charge de la constipation est spécifique chez ces patients; elle est associée à un traitement médical, à une rééducation périnéale et, dans certains cas, à une intervention chirurgicale (caecostomie [58] afin d'effectuer un lavage antérograde).

\section{Malformations médullaires et sacrées}

Une constipation peut avoir des origines neurologiques après une lésion médullaire (traumatismes, tumeurs, infections, etc.) [59], mais le diagnostic ne pose que peu de problèmes dans ces cas.

Des anomalies isolées du sacrum ou du filum terminale peuvent être révélées par une constipation. L'examen doit être centré sur la région sacrée (fossette, lipome, angiome, etc.). Après la réalisation d'une radiographie abdominale, le sacrum doit être étudié et une échographie chez les nouveau-nés ou une IRM doit compléter les examens radiologiques.

Par exemple, le syndrome de Currarino associe une tumeur présacrée (habituellement un tératome), à des anomalies sacrées (sacrum en cimeterre), et à des malformations anorectales (d'un anus quasiment normal à des malformations anorectales hautes) [60]. Les premiers signes cliniques peuvent être une constipation ou des difficultés urinaires.

En conclusion, la constipation est une cause fréquente de consultation au cours de l'enfance et ses étiologies sont très variables. Une évaluation attentive des antécédents médicaux et un examen clinique approfondi sont toutefois les meilleurs moyens de parvenir à un diagnostic. Aucun autre examen n'est nécessaire et toutes les explorations doivent être gérées en fonction des considérations cliniques.

\section{Références}

1 Constipation Guideline Committee of the North American Society for Pediatric Gastroenterology, Hepatology and Nutrition: Evaluation and treatment of constipation in infants and children: recommendations of the North American Society for Pediatric Gastroenterology, Hepatology and Nutrition. J Pediatr Gastroenterol Nutr 2006;43 e1-e13.

$\checkmark 2$ van den Berg MM, Benninga MA, Di Lorenzo C: Epidemiology of childhood constipation: a systematic review. Am J Gastroenterol 2006;101:2401-2409.

3 Khan S, Campo J, Bridge JA, et al: Long-term outcome of functional childhood constipation. Dig Dis Sci 2007;52:64-69.

-4 Singh SJ, Arbuckle S, Little D, et al: Mortality due to constipation and short-segment Hirschsprung's disease. Pediatr Surg Int 2004;20:889-891

5 Nurko S: What's the value of diagnostic tools in defecation disorders? J Pediatr Gastroenterol Nutr 2005;41(suppl 1):S53-S55.

-6 Drost J, Harris LA: Diagnosis and management of chronic constipation. JAAPA 2006; 19:24-29.
7 Biggs WS, Dery WH: Evaluation and treatment of constipation in infants and children. Am Fam Physician 2006;73:469-477.

8 Drossman DA, Dumitrascu DL: Rome III New standard for functional gastrointestinal disorders. J Gastrointestin Liver Dis 2006; 15:237-241.

-9 Rubin G, Dale A: Chronic constipation in children. BMJ 2006;333:1051-1055.

10 Remes-Troche JM, Rao SS: Diagnostic testing in patients with chronic constipation. Curr Gastroenterol Rep 2006;8:416-424.

11 Safder S, Rewalt M, Elitsur Y: Digital rectal examination and the primary care physicians: a lost art? Clin Pediatr 2006;45:411414.

12 Narbonne H, Paquis-Fluckinger V, Valero R, et al: Gastrointestinal tract symptoms in Maternally Inherited Diabetes and Deafness (MIDD). Diabetes Metab 2004;30:61-66.

13 Barrueto F Jr, Wang-Flores HH, Howland MA, et al: Acute vitamin D intoxication in a child. Pediatrics 2005;116:e453-e456.

14 Wu MJ, Chang CS, Cheng CH, et al: Colonic transit time in long-term dialysis patients. Am J Kidney Dis 2004;44:322-327.
15 Manz F, Wentz A: The importance of good hydration for the prevention of chronic diseases. Nutr Rev 2005;63:S2-S5.

16 Kawai M, Kubota A, Ida S, et al: Cow's milk allergy presenting Hirschsprung's diseasemimicking symptoms. Pediatr Surg Int 2005;21:850-852.

17 Rashid M, Cranney A, Zarkadas M, et al: Celiac disease: evaluation of the diagnosis and dietary compliance in Canadian children. Pediatrics 2005;116:e754-e759.

18 Kessmann J: Hirschsprung's disease: diagnosis and management. Am Fam Physician 2006;74:1319-1322.

19 Attie T, Salomon R, Amiel J, et al: Genetics of Hirschsprung disease (in French). CR Seances Soc Biol Fil 1996;190:549-556.

20 Lyonnet S, Bolino A, Pelet A, et al: A gene for Hirschsprung disease maps to the proximal long arm of chromosome 10. Nat Genet 1993; 4:346-350.

21 Blok S, Salomon R, Pelet A, et al: Segregation of three loci explains familial and population risks in Hirschsprung disease. Nat Genet 2002;31:89-93. 
-22 Amiel J, Lyonnet S: Hirschsprung disease, associated syndromes, and genetics: a review. J Med Genet 2001;38:729-739.

-23 Swenson O: Hirschsprung's disease: a review. Pediatrics 2002;109:914-918.

24 Reding R, de Ville de Goyet J, Gosseye S, et al: Hirschsprung's disease: a 20 -year experience. J Pediatr Surg 1997;32:1221-1225.

25 Polley TZ Jr, Coran AG, Wesley JR: A tenyear experience with ninety-two cases of Hirschsprung's disease. Including sixty-seven consecutive endorectal pull-through procedures. Ann Surg 1985;202:349-355.

26 Coran AG, Teitelbaum DH: Recent advances in the management of Hirschsprung's disease. Am J Surg 2000;180:382-387.

27 Stam H, Hartman EE, Deurloo JA, et al: Young adult patients with a history of pediatric disease: impact on course of life and transition into adulthood. J Adolesc Health 2006;39:4-13.

-28 Tsuji H, Spitz L, Kiely EM, et al: Management and long-term follow-up of infants with total colonic aganglionosis. J Pediatr Surg 1999; 34:158-162.

-29 Jasonni V, Martucciello G: Total colonic aganglionosis. Semin Pediatr Surg 1998;7: 174-180.

- 30 Escobar MA, Grosfeld JL, West KW, et al: Long-term outcomes in total colonic aganglionosis: a 32-year experience. J Pediatr Surg 2005;40:955-961.

- 31 Elhalaby EA, Teitelbaum DH, Coran AG, Heidelberger KP: Enterocolitis associated with Hirschsprung's disease: a clinical histopathological correlative study. J Pediatr Surg 1995;30:1023-1027.

32 Elhalaby EA, Coran AG, Blane CE, et al: Enterocolitis associated with Hirschsprung's disease: a clinical-radiological characterization based on 168 patients. J Pediatr Surg 1995;30:76-83.

- 33 Sarioglu A, Tanyel FC, Buyukpamukcu N, Hicsonmez A: Appendiceal perforation: a potentially lethal initial mode of presentation of Hirschsprung's disease. J Pediatr Surg 1997;32:123-124.

- 34 Teitelbaum DH, Coran AG: Enterocolitis. Semin Pediatr Surg 1998;7:162-169.

- 35 Courvoisier S, Piolat C, Durand C, et al: Pseudo-'isolated' intestinal perforation in a very low birth weight infant: exceptional presentation of Hirschsprung's disease (in French). Arch Pediatr 2006;13:1320-1322.
36 Arliss J, Holgersen LO: Neonatal appendiceal perforation and Hirschsprung's disease. J Pediatr Surg 1990;25:694-695.

37 Faure F, Panuel M, Petit PH, et al: Methodes d'explorations radiologiques de l'ano-rectum; in Le rectum pédiatrique. Monographie du college national de chirurgie pédiatrique. Montpellier, Sauramps Médical, 1996, pp 43-49.

38 Fotter R: Imaging of constipation in infants and children. Eur Radiol 1998;8:248-258.

39 Fondelli P, Martucciello G, Taccone A, Dodero P: The radiologic picture of total colonic aganglionosis (in Italian). Radiol Med (Torino) 1989;77:207-210.

-40 Fink RL, Roberts LJ, Scott M: The role of manometry, electromyography and radiology in the assessment of intractable constipation. Aust NZ J Surg 1992;62:959-964.

41 Meunier P, Marechal JM, Mollard P: Accuracy of the manometric diagnosis of Hirschsprung's disease. J Pediatr Surg 1978; 13:411-415.

42 Kapur RP: Can we stop looking? Immunohistochemistry and the diagnosis of Hirschsprung disease. Am J Clin Pathol 2006;126:9-12.

43 Matsuda H, Hirato J, Kuroiwa M, Nakazato Y: Histopathological and immunohistochemical study of the enteric innervations among various types of aganglionoses including isolated and syndromic Hirschsprung disease. Neuropathology 2006;26:823.

-44 Hartman EE, Sprangers MA, Visser MR, et al: Hirschsprung's disease: healthcare meets the needs. J Pediatr Surg 2006;41:14201424.

45 Catto-Smith AG, Trajanovska M, Taylor RG: Long-term continence in patients with Hirschsprung's disease and Down syndrome. J Gastroenterol Hepatol 2006;21: 748-753.

46 Menezes M, Puri P: Long-term outcome of patients with enterocolitis complicating Hirschsprung's disease. Pediatr Surg Int 2006;22:316-318.

47 Hyman PE: Defecation disorders after surgery for Hirschsprung's disease. J Pediatr Gastroenterol Nutr 2005;41(suppl 1):S62S63.

48 Saleh W, Rasheed K, Mohaidly MA, et al: Management of Hirschsprung's disease: a comparison of Soave's and Duhamel's pullthrough methods. Pediatr Surg Int 2004;20: 590-593.
49 Minford JL, Ram A, Turnock RR, et al: Comparison of functional outcomes of Duhamel and transanal endorectal coloanal anastomosis for Hirschsprung's disease. J Pediatr Surg 2004;39:161-165.

- 50 Dasgupta R, Langer JC: Transanal pullthrough for Hirschsprung disease. Semin Pediatr Surg 2005;14:64-71

51 Podevin G, Lardy H, Azzis O, et al: Technical problems and complications of a transanal pull-through for Hirschsprung's disease. Eur J Pediatr Surg 2006;16:104-108.

52 Yamauchi K, Kubota A, Usui N, et al: Benign transient non-organic ileus of neonates. Eur J Pediatr Surg 2002;12:168-174.

53 Cowles RA, Berdon WE, Holt PD, et al: Neonatal intestinal obstruction simulating meconium ileus in infants with long-segment intestinal aganglionosis: radiographic findings that prompt the need for rectal biopsy. Pediatr Radiol 2006;36:133-137.

54 Joly F, Amiot A, Coffin B, et al: Chronic intestinal pseudo-obstruction (in French). Gastroenterol Clin Biol 2006;30:975-985.

55 Meier-Ruge WA, Bruder E: Pathology of chronic constipation in pediatric and adult coloproctology. Pathobiology 2005;72:1-102.

56 Hartman EE, Oort FJ, Aronson DC, et al: Critical factors affecting quality of life of adult patients with anorectal malformations or Hirschsprung's disease. Am J Gastroenterol 2004;99:907-913.

57 Hamid CH, Holland AJ, Martin HC: Longterm outcome of anorectal malformations: the patient perspective. Pediatr Surg Int 2006;Epub ahead of print.

58 Yagmurlu A, Harmon CM, Georgeson KE: Laparoscopic cecostomy button placement for the management of fecal incontinence in children with Hirschsprung's disease and anorectal anomalies. Surg Endosc 2006;20: 624-627.

59 Valles M, Vidal J, Clave P, Mearin F: Bowel dysfunction in patients with motor complete spinal cord injury: clinical, neurological, and pathophysiological associations. Am J Gastroenterol 2006;101:2290-2299.

-60 Kilickesmez O, Gol IH, Uzun M, Oruk C: Complete familial Currarino triad in association with Hirschsprung's disease: magnetic resonance imaging features and the spectrum of anorectal malformations. Acta Radiol 2006;47:422-426. 